\title{
The Piezoelectric Constant for Disperse Systems
}

\author{
Munehiro DATE \\ The Institute of Physical and Chemical Research, \\ Wako-shi, Saitama 351, Japan.
}

(Received June 16, 1975)

\begin{abstract}
A mathematical expression is derived for the piezoelectric constant of a disperse system in which piezoelectric, anisotropic, spherical particles are uniformly dispersed in a nonpiezoelectric, isotropic, continuous medium.

For the case when the sphere has the face-shear-type piezoelectricity and the system is incompressible, the piezoelectric strain constant $d_{14}$ for the disperse system is given as

$$
d_{14}=d_{14}^{\mathrm{II}} \frac{3}{2+\varepsilon^{\mathrm{II}} / \varepsilon^{\mathrm{I}}+\left(1-\varepsilon^{\mathrm{II}} / \varepsilon^{\mathrm{I}}\right) \phi} \cdot \frac{5}{2+3 G^{\mathrm{I}} / G^{\mathrm{II}}+3\left(1-G^{\mathrm{I}} / G^{\mathrm{II}}\right) \phi} \phi
$$

where $\phi$ is the volume fraction of spheres, $d_{14}^{\mathrm{II}}$ the piezoelectric strain constant of the sphere, $\varepsilon$ the dielectric constant, $G$ the elastic constant, and the superscripts I and II refer to the medium and sphere, respectively.

For the case when the sphere is extremely rigid and has length-expansion and thickness-expansion types of piezoelectricity, the piezoelectric strain constants $d_{31}, d_{32}$, $d_{33}$ under the approximation $\phi \ll 1$ are given as
\end{abstract}

$$
\left[\begin{array}{l}
d_{31} \\
d_{32} \\
d_{33}
\end{array}\right]=\frac{3 \phi}{2+\frac{\varepsilon_{3}^{\mathrm{II}}}{\varepsilon^{\mathrm{I}}}} \frac{1+\frac{4}{3} \frac{G^{\mathrm{I}}}{K^{\mathrm{I}}}}{2+\frac{4 G^{\mathrm{I}}}{K^{\mathrm{I}}}}\left[\begin{array}{ccc}
\alpha+4 & \alpha-1 & \alpha-1 \\
\alpha-1 & \alpha+4 & \alpha-1 \\
\alpha-1 & \alpha-1 & \alpha+4
\end{array}\right]\left[\begin{array}{l}
d_{31}^{\mathrm{II}} \\
d_{32}^{\mathrm{II}} \\
d_{33}^{\mathrm{II}}
\end{array}\right]
$$

where $K^{\mathrm{I}}$ is the bulk modulus of the medium and $\alpha=4 G^{\mathrm{I}} / 3 K^{\mathrm{I}}$.

KEY WORDS Piezoelectric Constant / Spherical Disperse System /

Electromechanical Coupling / Piezoelectric Dispersion / Piezoelectric

Polymers / Elasticity / Dielectric Properties /

The piezoelectric constants of polymers depend upon the temperature and frequency of measurement and are expressed by complex quantities, like the elastic and dielectric constants. ${ }^{1}$ The intrinsic piezoelectricity of polymers is believed to be caused by the rotation of dipoles under the action of the mechanical or electrical field in the oriented or crystallized regions of the polymer. These piezoelectric regions are generally imbedded in unoriented nonpiezoelectric regions. The origin of the piezoelectric dispersion is therefore to be explained on the basis of the binary structure for the polymer.

Mechanical and electrical equivalent models that explain the piezoelectric dispersion were previously proposed by Fukada and Date. ${ }^{2}$ Hayakawa and $\mathrm{Wada}^{3}$ have recently derived a systematic theory for polymer piezoelectricity. They presented a mechanical two phase model that elucidated the relation between the phase angle of the piezoelectric constant and the mechanical relaxation of the nonpiezoelectric phase surrounding the piezoelectric phase.

The simplest model for a two phase system is a spherical disperse system in which piezoelectric spherical particles are uniformly dispersed in a nonpiezoelectric continuous medium. We shall derive here an expression for the piezoelectric constant of this spherical dispersion model.

\section{THEORETICAL EQUATIONS}

In the present paper a mathematical expession for the piezoelectric constant for a spherical disperse system is derived. First, we make the following assumptions.

(1) The relations between mechanical and elec- 
trical quantities are all linear.

(2) The non-piezoelectric medium (phase I) is isotropic.

(3) In the elastic deformation, no slip occurs on the surface of the spheres, and the strain in the dispersed spheres is uniform when the external macroscopic strain of the system is uniform.

(4) The anisotropic axes of the dispersed spheres are oriented in the same directions.

The fundamental piezoelectric equations for the disperse system are given as follows.

$$
\left[\begin{array}{c}
X_{i} \\
D_{l}
\end{array}\right]=\left[\begin{array}{cc}
C_{i j} & -e_{k i} \\
4 \pi e_{l j} & \varepsilon_{l k}
\end{array}\right]\left[\begin{array}{c}
x_{j} \\
E_{k}
\end{array}\right]
$$

where $X_{i}$ and $x_{j}$ are the stress and strain respectively, $D_{l}$ the electric displacement, $E_{l c}$ the electric field, $e_{k i}$ the piezoelectric stress constant, $C_{i j}$ the elastic constant, and $\varepsilon_{l k}$ the dielectric constant.

In principle, these quantities should be derived from a free energy $F_{0}(x, E)$ of the system, with $\boldsymbol{x}$ and $\boldsymbol{E}$ standing for the strain and electric field of the system respectively. In order to obtain the relations among these elastic and dielectric quantities of the medium and the dispersed spheres, we will define a characteristic function, $F(\boldsymbol{x}, \boldsymbol{E}$, $\left.\boldsymbol{x}^{\mathrm{II}}, \boldsymbol{E}^{\mathrm{II}}\right)$, where $\boldsymbol{x}^{\mathrm{II}}$ and $\boldsymbol{E}^{\mathrm{II}}$ are strain and electric field of the dispersed spheres respectively.

Two virtual parameters, $\boldsymbol{f}^{\nabla}$ and $q^{\nabla}$, are introduced to meet the requirement that $\boldsymbol{x}^{\mathrm{II}}$ and $\boldsymbol{E}^{\mathrm{II}}$ are independent variables in this characteristic function. $\boldsymbol{f}^{\nabla}$ and $q^{\nabla}$ are the virtual force per unit area and the virtual true charge density added to the boundary of the dispersed sphere, respectively. Since in the actual system, $f^{\vee}$ and $q^{\nabla}$ must be equal to zero, we have

$$
F_{0}(\boldsymbol{x}, \boldsymbol{E})=F\left(\boldsymbol{x}, \boldsymbol{E}, \boldsymbol{x}^{\mathrm{II}}, E^{\mathrm{II}}\right)_{f^{\vee}=0, q^{\vee}=0}
$$

First we shall try to find the functional form of $F$ when the virtual force $f^{\nabla}$ and the virtual charge $q^{\nabla}$ are present in dispersed spheres.

Mechanical and electrical work, $\mathrm{d} W_{\mathrm{m}}$ and $\mathrm{d} W_{\mathrm{e}}$, added to a unit volume of the system by the differentials $\mathrm{d} x$ and $\mathrm{d} D$ are written as follows

and

$$
\mathrm{d} W_{\mathrm{m}}=X_{i} \mathrm{~d} x_{i}
$$

$$
\mathrm{d} W_{\mathrm{e}}=\frac{1}{4 \pi} E_{i} \mathrm{~d} D_{i}
$$

The virtual mechanical work, $\mathrm{d} W_{\mathrm{m}}{ }^{ }$, by the differential $\mathrm{d} \boldsymbol{l}$ is written as follows:

$$
\mathrm{d} W_{\mathrm{m}}{ }^{\nabla}=\sum_{k} \int_{S_{k}} \boldsymbol{f}^{\nabla} \mathrm{d} \boldsymbol{l} \mathrm{d} S_{k}
$$

where $l$ is the real displacement on the surface of the sphere and $S_{k}$ the surface area of the $k$-th sphere.

If the origin of coordinates is taken at the center of a sphere $k$, then $\boldsymbol{f}^{\nabla}$ and $\boldsymbol{l}$ can be written as follows

and

$$
\boldsymbol{f}^{\nabla}=\boldsymbol{X}^{\nabla} \boldsymbol{n}
$$

$$
\boldsymbol{l}=\boldsymbol{x}^{\mathrm{II}} \boldsymbol{n} \boldsymbol{r}_{k}
$$

where $\boldsymbol{X}^{\mathrm{v}}$ and $\boldsymbol{x}^{\mathrm{II}}$ are the virtual stress and the strain in the sphere, respectively, $n$ the unit vector normal to the surface of the sphere, and $r_{k}$ the radius of the $k$-th sphere. ${ }^{*}$ When eq 5 , 6 , and 7 are combined, one obtains

$$
\begin{aligned}
\mathrm{d} W_{\mathrm{m}}{ }^{\nabla} & =\sum_{k} \int_{S_{k}}\left(\boldsymbol{X}^{\nabla} \boldsymbol{n}\right) \cdot\left(\mathrm{d} \boldsymbol{x}^{\mathrm{II}} \boldsymbol{n} r_{k}\right) \mathrm{d} S_{k} \\
& =\sum_{k} \boldsymbol{X}^{\mathrm{v}} \mathrm{d} \boldsymbol{x}^{\mathrm{II}} V_{k} \\
& =\phi \boldsymbol{X}^{\mathrm{v}} \mathrm{d} \boldsymbol{x}^{\mathrm{II}}
\end{aligned}
$$

where $\boldsymbol{X}^{\mathrm{v}} \mathrm{d} \boldsymbol{x}^{\mathrm{II}}$ denotes $\sum_{i}^{3} \sum_{j}^{3} X_{i j}^{\mathrm{V}} \mathrm{d} x_{i j}^{\mathrm{II}}, \quad V_{k}$ the volume of $k$-th sphere, and $\phi$ the volume fraction of the spheres, $\sum_{k} V_{k}$.

The virtural electrical work, $\mathrm{d} W_{\mathrm{e}}^{\nabla}$, that is, the work done by the virtual charge $\mathrm{d} q^{v}$ when being transfered from infinity to the surface of the sphere, is given as

$$
\mathrm{d} W_{\mathrm{e}}^{\nabla}=\sum_{k} \int \mathrm{d} q^{\nabla} \psi \mathrm{d} S_{k}
$$

where $\psi$ is the electric potential on the surface of the sphere. The virtual electric displacement $D^{\nabla}$ is defined by

$$
\mathrm{d} q^{\nabla}=-\frac{1}{4 \pi} n \mathrm{~d} \boldsymbol{D}^{\nabla}
$$

Substituting eq 10 to eq 9 , one finds

$$
\begin{aligned}
\mathrm{d} W_{\mathrm{e}}^{\nabla} & =-\frac{1}{4 \pi} \sum_{k} \int_{S_{k}}\left(\mathrm{~d} \boldsymbol{D}^{\nabla} \boldsymbol{n}\right) \cdot\left(\operatorname{grad} \phi \boldsymbol{n} r_{k}\right) \mathrm{d} S_{k} \\
& =\frac{1}{4 \pi} \sum_{k} r_{k} \int_{S_{k}}\left(\mathrm{~d} \boldsymbol{D}^{\vee} \boldsymbol{n}\right) \cdot\left(\boldsymbol{E}^{\mathrm{II}} \boldsymbol{n}\right) \mathrm{d} S_{k}
\end{aligned}
$$

* In this paper, the stress and strain are mostly expressed by matrix notation. However, in eq 6 , 7 , and $8, \boldsymbol{X}^{\vee}$ and $\boldsymbol{x}^{\mathrm{II}}$ are expressed in a second rank tensor respectively. 


$$
=\frac{1}{4 \pi} \phi E^{\mathrm{II}} \mathrm{d} D^{\nabla}
$$

Therefore, the differential $\mathrm{d} U$ of the internal energy, which takes into account both the virtual mechanical and electrical work, is given by

$$
\begin{gathered}
\mathrm{d} U=T \mathrm{~d} S+\mathrm{d} W_{\mathrm{m}}+\mathrm{d} W_{\mathrm{e}}+\mathrm{d} W_{\mathrm{m}}{ }^{\nabla}+\mathrm{d} W_{\mathrm{e}}^{\nabla} \\
=T \mathrm{~d} S+\boldsymbol{X} \mathrm{d} \boldsymbol{x}+\frac{1}{4 \pi} \boldsymbol{E} \mathrm{d} \boldsymbol{D}+\phi \boldsymbol{X}^{\nabla} \mathrm{d} \boldsymbol{x}^{\mathrm{II}} \\
+\frac{1}{4 \pi} \phi \boldsymbol{E}^{\mathrm{II}} \mathrm{d} \boldsymbol{D}^{\nabla}
\end{gathered}
$$

where $S$ is the entropy and $T$ the temperature.

Now let us define a characteristic function $F\left(\boldsymbol{x}, E, \boldsymbol{x}^{\mathrm{II}}, \boldsymbol{E}^{\mathrm{II}}\right)$ as follows

$$
F=U-T S-\frac{1}{4 \pi} E D-\frac{1}{4 \pi} \phi E^{\mathrm{II}} D^{\nabla}
$$

From this definition, one obtains the total differential of $F$.

$$
\begin{aligned}
\mathrm{d} F= & -S \mathrm{~d} T+\boldsymbol{X} \mathrm{d} \boldsymbol{x}-\frac{1}{4 \pi} D \mathrm{~d} \boldsymbol{E}+\phi \boldsymbol{X}^{\mathrm{V}} \mathrm{d} \boldsymbol{x}^{\mathrm{II}} \\
& -\frac{1}{4 \pi} \phi \boldsymbol{D}^{\mathrm{v}} \mathrm{d} \boldsymbol{E}^{\mathrm{II}} .
\end{aligned}
$$

Since we consider here an isothermal process, $S \mathrm{~d} T$ is equal to zero.

From this characteristic function, the following relations are obtained

$$
\begin{gathered}
X_{\mathrm{i}}=\left[\frac{\partial F}{\partial x_{i}}\right]_{E, x^{\mathrm{II}}, E \mathrm{II}} \\
D_{\mathrm{i}}=-4 \pi\left[\frac{\partial F}{\partial E_{i}}\right]_{x, x \mathrm{II}, E^{\mathrm{II}}} \\
X_{1}^{\nabla}=\frac{1}{\phi}\left[\frac{\partial F}{\partial x_{i}^{\mathrm{II}}}\right]_{x, E, E} \mathrm{II} \\
D_{\mathrm{I}}^{\nabla}=-\frac{4 \pi}{\phi}\left[\frac{\partial F}{\partial E_{i}^{\mathrm{II}}}\right]_{x, E, x} \mathrm{II}
\end{gathered}
$$

Differentials $\mathrm{d} X_{i}, \mathrm{~d} D_{i}, \mathrm{~d} X_{i}{ }^{\nabla}$, and $\mathrm{d} D_{i}{ }^{\nabla}$ can be expressed by the independent variables, $x_{j}, E_{j}$, $x_{j}{ }^{\mathrm{II}}$, and $E_{j}{ }^{\mathrm{II}}$, as in the following example

$$
\begin{aligned}
\mathrm{d} X_{i}= & \frac{\partial X_{i}}{\partial x_{j}} \mathrm{~d} x_{j}+\frac{\partial X_{i}}{\partial E_{j}} \mathrm{~d} E_{j}+\frac{\partial X_{i}}{\partial x_{j}{ }^{\mathrm{II}}} \mathrm{d} x_{j}{ }^{\mathrm{II}}+\frac{\partial X_{i}}{\partial E_{j}{ }^{\mathrm{II}}} \mathrm{d} E_{j}{ }^{\mathrm{II}} \\
= & \frac{\partial^{2} F}{\partial x_{i} \partial x_{j}} \mathrm{~d} x_{j}+\frac{\partial^{2} F}{\partial x_{i} \partial E_{j}} \mathrm{~d} E_{j} \\
& +\frac{\partial^{2} F}{\partial x_{i} \partial x_{j}{ }^{\mathrm{II}}} \mathrm{d} x_{j}{ }^{\mathrm{II}}+\frac{\partial^{2} F}{\partial x_{i} \partial E_{j}{ }^{\mathrm{II}}} \mathrm{d} E_{j}{ }^{\mathrm{II}}
\end{aligned}
$$

For the differentials $\mathrm{d} D_{i}, \mathrm{~d} X_{i}{ }^{\nabla}$, and $\mathrm{d} D_{i}{ }^{\mathrm{v}}$, one obtains similar equations. For simplicity the differential operator $\mathrm{d}$ will be omitted, for example, $\mathrm{d} X$ will be written as $X$. Now, the derivatives $\left[\partial D_{i} / \partial x_{j}\right]_{E, \boldsymbol{x}} \mathrm{II}, \boldsymbol{E}^{\mathrm{II}},\left[\partial D_{i}^{\nabla} / \partial x_{j}\right]_{\boldsymbol{E}, \boldsymbol{x}} \mathrm{II}, \boldsymbol{E} \mathrm{II}$, $\left[\partial X_{i} / \partial E_{j}\right]_{x, x} \mathrm{II}, \boldsymbol{E} \mathrm{II}$, and $\left[\partial X_{i}^{\mathrm{V}} / \partial E_{j}\right]_{x, \boldsymbol{x}} \mathrm{II}, E^{\mathrm{II}}$, are equal to zero because the medium is nonpiezoelectric and $\boldsymbol{x}^{\mathrm{II}}$ and $\boldsymbol{E}^{\mathrm{II}}$ are kept constant in evaluating these partial derivatives. Similarly, $\left[\partial X_{i} / \partial E_{j}{ }^{\mathrm{II}}\right]_{x, E, x} \mathrm{II}$ and $\left[\partial D_{i} / \partial x_{j}{ }^{\mathrm{II}}\right]_{x, E, E} \mathrm{II}$ are also equal to zero. From eq $15,16,17$, and 18 , the following reciprocal relations can be obtained

$$
\begin{aligned}
& \phi\left[\frac{\partial X_{i}{ }^{\mathrm{v}}}{\partial x_{j}}\right]_{E, x \mathrm{II}, E \mathrm{II}}=\left[\frac{\partial X_{j}}{\partial x_{i}^{\mathrm{II}}}\right]_{x, E, E} \\
& \phi\left[\frac{\partial D_{i}{ }^{\mathrm{V}}}{\partial E_{i}}\right]_{x, \boldsymbol{x} \mathrm{II}, \boldsymbol{E}^{\mathrm{II}}}=\left[\frac{\partial \boldsymbol{D}_{i}}{\partial E_{i}^{\mathrm{II}}}\right]_{\boldsymbol{x}, \boldsymbol{E}, \boldsymbol{x} \mathrm{II}} \\
& {\left[\frac{\partial D_{i}{ }^{\mathrm{V}}}{\partial x_{j}^{\mathrm{II}}}\right]_{x, E, E \mathrm{II}}=-4 \pi\left[\frac{\partial X_{j}^{\mathrm{V}}}{\partial E_{i}^{\mathrm{II}}}\right]_{x, E, x \mathrm{II}}}
\end{aligned}
$$

with these relations, eq 19 and other similar equations are simplified as follows

$$
\left[\begin{array}{c}
X_{i} \\
D_{i} \\
X_{i}{ }^{\mathrm{v}} \\
D_{i}{ }^{\mathrm{v}}
\end{array}\right]=\left[\begin{array}{cccc}
C_{i j}^{\mathrm{r}} & 0 & -\phi C_{i j}^{\mathrm{m}} & 0 \\
0 & \varepsilon^{\mathrm{c}} \delta_{i j} & 0 & -\phi \varepsilon^{\mathrm{m}} \delta_{i j} \\
-C_{i j}^{\mathrm{m}} & 0 & C_{i j}^{\mathrm{II}} & -e_{j i}^{\mathrm{II}} \\
0 & -\varepsilon^{\mathrm{m}} \delta_{i j} & 4 \pi e_{i j}^{\mathrm{II}} & \varepsilon_{i j}^{\mathrm{II} \mathrm{c}}
\end{array}\right]\left[\begin{array}{c}
x_{j} \\
E_{j} \\
x_{j}^{\mathrm{II}} \\
E_{j}{ }^{\mathrm{II}}
\end{array}\right]
$$

where $C_{i j}^{\mathrm{r}}$ and $\varepsilon^{\mathrm{c}}$ are the elastic and dielectric constant of the disperse system under the condition that the dispersed spheres are rigid and conductive, $C_{i j}^{\mathrm{II}}$ and $\varepsilon_{i j}^{\mathrm{II}_{\mathrm{c}}}$ the equivalent elastic and dielectric constant of the spheres under the condition that the surfaces of the system are clamped and shorted, $C_{i j}^{\mathrm{m}}$ and $\varepsilon^{\mathrm{m}}$ the mutual elastic and dielectric constants between the sphere and the whole system, and $e_{i j}^{\mathrm{II}}$ the piezoelectric constant of the dispersed spheres. It is seen from relations such as eq 19, that the subscripts $i j$ in $C_{i j}^{\mathrm{r}}, \varepsilon_{i j}^{\mathrm{c}}, C_{i j}^{\mathrm{II}}$, and $\varepsilon_{i j}^{\mathrm{II}}$ are commutative. The subscript $i j$ in $C_{i j}^{\mathrm{m}}$ is also commutative, because the medium is isotropic.

More exact definitions for these coefficients are given in the appendix. Among the seven coefficients in eq $23, C^{r}$, the elastic constant of the dispersed system of rigid spheres and $\varepsilon^{c}$, the dielectric constant of the dispersed system of conductive spheres have been already analytically derived in terms of the elastic and dielectric constants of the medium and the sphere, respec- 
The Piezoelectric Constant for Disperse Systems

tively. ${ }^{4,5}$ Here we shall find the expressions of other coefficients, $\boldsymbol{C}^{\mathrm{m}}, \boldsymbol{C}^{\mathrm{II}_{\mathrm{r}}}, \varepsilon^{\mathrm{m}}$, and $\varepsilon^{\mathrm{II}_{\mathrm{c}}}$.

If we assume that $\boldsymbol{x}^{\mathrm{II}}$ is equal to $\boldsymbol{x}$, though the elastic constant of the medium is different from that of the spheres, we should have

and

$$
X_{i}=C_{i j}^{\mathrm{I}} x_{j}
$$

$$
X_{i}^{\mathrm{v}}=\left(C_{i j}^{\mathrm{II}}-C_{i j}^{\mathrm{I}}\right) x_{j}
$$

These equations imply that the binary system cannot be distinguished from a single phase system of medium only and a virtual force $X_{i}{ }^{\mathrm{V}}$ is required to balance the excess force on the boundary between the sphere and the medium, where $C^{\mathrm{I}}$ and $C^{\mathrm{II}}$ are the elastic constants of the medium and of the dispersed sphere, respectively.

From eq 23, putting $x_{j}{ }^{\mathrm{II}}=x_{j}$, we obtain

$$
X_{i}=C_{i j}^{\mathrm{r}} x_{j}-\phi C_{i j}^{\mathrm{m}} x_{j}
$$

Substituting eq 24 into eq 26 , we obtain

$$
C_{i j}^{\mathrm{m}}=\frac{1}{\phi}\left(C_{i j}^{\mathrm{r}}-C_{i j}^{\mathrm{I}}\right)
$$

From eq 23, putting $E_{j}{ }^{\mathrm{II}}=0$ and $x_{j}{ }^{\mathrm{II}}=x_{j}$, we obtain

$$
X_{i}^{\nabla}=-C_{i j}^{\mathrm{m}} x_{j}+C_{i j}^{\mathrm{II} r} x_{j}
$$

Then combining eq 25,27 , and 28 we obtain

$$
C_{i j}^{\mathrm{II}}=C_{i j}^{\mathrm{II}}+\frac{1}{\phi}\left(C_{i j}^{\mathrm{r}}-C_{i j}^{\mathrm{I}}\right)-C_{i j}^{\mathrm{I}}
$$

If we assume that $E^{\text {II }}$ equals to $E$, though the dielectric constant of the medium is different from that of the sphere, the following relations must be satisfied

and

$$
D_{i}=\varepsilon^{\mathrm{I}} E_{i}
$$

$$
D_{i}{ }^{\nabla}=\left(\varepsilon_{i j}^{\mathrm{II}}-\varepsilon^{\mathrm{I}} \delta_{i j}\right) E_{j}, \quad \delta_{i j}= \begin{cases}1 & i=j \\ 0 & i \neq j\end{cases}
$$

where $\varepsilon^{\mathrm{I}}$ and $\varepsilon^{\mathrm{II}}$ are the dielectric constants of the medium and the spheres respectively. From eq 23 , putting $E_{j}{ }^{\mathrm{II}}=E_{j}$,

and

$$
D_{i}=\varepsilon^{\mathrm{c}} E_{i}-\phi \varepsilon^{\mathrm{m}} E_{i}
$$

$$
D_{i}{ }^{\nabla}=-\varepsilon^{\mathrm{m}} \delta_{i j} E_{j}+\varepsilon_{i j}^{1 \mathrm{II}} E_{j}
$$

Since the medium is an isotropic body, $\varepsilon^{c}$ and $\varepsilon^{\mathrm{m}}$ are scalars. From eq 30 and 32 ,

$$
\varepsilon^{\mathbf{m}}=\frac{1}{\phi}\left(\varepsilon^{\mathrm{c}}-\varepsilon^{\mathbf{I}}\right)
$$

Combining eq 31,33 , and 34 , we obtain

$$
\varepsilon_{i j}^{\mathrm{II}}=\varepsilon_{i j}^{\mathrm{II}}+\left[\frac{1}{\phi}\left(\varepsilon^{\mathrm{c}}-\varepsilon^{\mathrm{I}}\right)-\varepsilon^{\mathrm{I}}\right] \delta_{i j}
$$

Now we shall proceed to obtain more concrete expressions for the coefficients in eq 23. The elastic tensor for any isotropic body is a function of two independent components, $C$ and $\lambda$, explicitly,

$$
\left(C_{i j}\right)=\left[\begin{array}{cccccc}
C & \lambda & \lambda & 0 & 0 & 0 \\
\lambda & C & \lambda & 0 & 0 & 0 \\
\lambda & \lambda & C & 0 & 0 & 0 \\
0 & 0 & 0 & G & 0 & 0 \\
0 & 0 & 0 & 0 & G & 0 \\
0 & 0 & 0 & 0 & 0 & G
\end{array}\right]
$$

where the shear modulus $G$ and the bulk modulus $K$ are given as $G=(C-\lambda) / 2$ and $K=(C+2 \lambda) / 3$, respectively.

For the isotropic spherical dispersion system, $G$ and $K$ have been derived as follows ${ }^{4}$

$$
\begin{gathered}
G=G^{\mathrm{I}} \frac{1+\frac{\left(G^{\mathrm{II}}-G^{\mathrm{I}}\right)\left(9 K^{\mathrm{I}}+8 G^{\mathrm{I}}\right)}{6 G^{\mathrm{II}}\left(K^{\mathrm{I}}+2 G^{\mathrm{I}}\right)+G^{\mathrm{I}}\left(9 K^{\mathrm{I}}+8 G^{\mathrm{I}}\right)} \phi}{1-\frac{6\left(G^{\mathrm{II}}-G^{\mathrm{I}}\right)\left(K^{\mathrm{I}}+2 G^{\mathrm{I}}\right)}{6 G^{\mathrm{II}}\left(K^{\mathrm{I}}+2 G^{\mathrm{I}}\right)+G^{\mathrm{I}}\left(9 K^{\mathrm{I}}+8 G^{\mathrm{I}}\right)} \phi} \\
K=K^{\mathrm{I}}+\left(K^{\mathrm{II}}-K^{\mathrm{I}}\right) \frac{K^{\mathrm{I}}+\frac{4}{3} G^{\mathrm{I}}}{K^{\mathrm{II}}+\frac{4}{3} G^{\mathrm{I}}} \phi
\end{gathered}
$$

where superscripts I and II refer to the medium and the dispersed phase, respectively.

Now we consider the case in which the dispersed spheres are perfectly rigid $\left(G^{\mathrm{II}}=K^{\mathrm{II}}=\infty\right)$. Using the relations $G=(C-\lambda) / 2$ and $K=(C+2 \lambda) /$ 3 , from eq 37 and 38 , the elastic constant of the dispersed system of rigid spheres is given as

$$
C_{i j}^{\mathrm{I}}= \begin{cases}C^{\mathrm{I}}+\left(C^{\mathrm{I}}+\alpha\right) \phi & (i=j \text { and } i, j=1,2,3) \\ \lambda^{\mathrm{I}}+\left(C^{\mathrm{I}}-\frac{\alpha}{2}\right) \phi & (i \neq j \text { and } i, j=1,2,3) \\ \frac{C^{\mathrm{I}}-\lambda^{\mathrm{I}}}{2}+\frac{3}{4} \alpha \phi & (i=j \text { and } i, j=4,5,6) \\ 0 & (i \neq j \text { and } i, j=4,5,6)\end{cases}
$$

where $\alpha=\left[5\left(C^{\mathrm{I}}-\lambda^{\mathrm{I}}\right) /(1-\phi)\right]\left[C^{\mathrm{I}} /\left(4 C^{\mathrm{I}}-\lambda^{\mathrm{I}}\right)\right]$.

Substituting eq 39 into eq 27 and 29 , we obtain

$$
C_{i j}^{\mathrm{m}}= \begin{cases}C^{\mathrm{I}}+\alpha & (i=j \text { and } i, j=1,2,3) \\ C^{\mathrm{I}}-\frac{\alpha}{2} & (i \neq j \text { and } i, j=1,2,3) \\ \frac{4}{3} \alpha & (i=j \text { and } i, j=4,5,6) \\ 0 & (i \neq j \text { and } i, j=4,5,6)\end{cases}
$$


where $\alpha=\left[5\left(C^{\mathrm{I}}-\lambda^{\mathbf{I}}\right) /(1-\phi)\right]\left[C^{\mathbf{I}} /\left(4 C^{\mathrm{I}}-\lambda^{\mathrm{I}}\right)\right]$.

$$
C_{i j}^{\mathrm{II} \mathrm{r}}= \begin{cases}C_{i j}^{\mathrm{II}}+\alpha & (i=j \text { and } i, j=1,2,3) \\ C_{i j}^{\mathrm{II}}+C^{\mathrm{I}}-\lambda^{\mathrm{I}}-\frac{\alpha}{2} & (i \neq j \text { and } i, j=1,2,3) \\ C_{i j}^{\mathrm{II}}-\frac{C^{\mathrm{I}}-\lambda^{\mathrm{I}}}{2}+\frac{3}{4} & (i=j \text { and } i, j=4,5,6) \\ C_{i j}^{\mathrm{II}} & (i \neq j \text { and } i, j=4,5,6)\end{cases}
$$

where $\alpha=\left[5\left(C^{\mathrm{I}}-\lambda^{\mathrm{I}}\right) /(1-\phi)\right]\left[C^{\mathrm{I}} /\left(4 C^{\mathrm{I}}-\lambda^{\mathrm{I}}\right)\right]$.

Equation 39,40 , and 41 can also be represented in terms of $G^{\mathrm{I}}, G^{\mathrm{II}}, K^{\mathrm{I}}$, and $K^{\mathrm{II}}$, using the relations $C=K+(4 / 3) G$, and $\lambda=K-(2 / 3) G$.

The dielectric constant of the dispersed system has been given $a^{5}$

$$
\varepsilon=\varepsilon^{\mathrm{I}}\left[1+\frac{3\left(\varepsilon^{\mathrm{II}}-\varepsilon^{\mathrm{I}}\right) \phi}{2 \varepsilon^{\mathrm{I}}+\varepsilon^{\mathrm{II}}-\left(\varepsilon^{\mathrm{II}}-\varepsilon^{\mathrm{I}}\right) \phi}\right]
$$

If the spheres are electrically conductive $\left(\varepsilon^{\text {II }}=\infty\right)$, we have

$$
\varepsilon^{\mathrm{c}}=\varepsilon^{\mathrm{I}}\left(1+\frac{3 \phi}{1-\phi}\right)
$$

Substituting eq 43 into eq 34 and 35 , we obtain

$$
\varepsilon^{\mathrm{m}}=\frac{3}{1-\phi} \varepsilon^{\mathrm{I}}
$$

and

$$
\varepsilon_{i j}^{\mathrm{I} \mathrm{C}}=\varepsilon_{i j}^{\mathrm{II}}+\varepsilon^{\mathrm{I}}\left(\frac{3}{1-\phi}-1\right) \delta_{i j}, \quad \delta_{i j}= \begin{cases}1 & i=j \\ 0 & i \neq j\end{cases}
$$

Using eq 39, 40, 41, 44, and 45, all components of the matrix of eq 23 can be represented by the material constants of the medium and the dispersed spheres, i.e., $C_{i j}^{\mathrm{I}}, \varepsilon_{i j}^{\mathrm{I}}, C_{i j}^{\mathrm{II}}, \varepsilon_{i j}^{\mathrm{II}}$, and $e_{i j}^{\mathrm{II}}$.

Replacing the independent variables $x_{j}{ }^{\mathrm{II}}$ and $E_{j}{ }^{\mathrm{II}}$ in eq 23 by $X_{i}{ }^{\nabla}$ and $D_{i}{ }^{\nabla}$, setting $X_{i}{ }^{\mathrm{v}}$ and $D_{i}{ }^{\mathrm{v}}$ equal to zero, which is the case for the actual system, we obtain

$$
\left[\begin{array}{c}
X_{i} \\
D_{i} \\
x_{i}^{\mathrm{II}} \\
E_{i}^{\mathrm{II}}
\end{array}\right]=\left[\begin{array}{l}
M_{1} \\
M_{2}
\end{array}\right]\left[\begin{array}{c}
x_{j} \\
E_{j}
\end{array}\right]
$$

When we compare eq 1 with eq 46 , the submatrix $\boldsymbol{M}_{1}$ is equal to the matrix of eq 1 . Since the components of $\boldsymbol{M}_{1}$ are given in terms of $C_{i j}^{\mathrm{I}}, C_{i j}^{\mathrm{II}}, \varepsilon_{i j}^{\mathrm{I}}$, and $\varepsilon_{i j}^{\mathrm{II}}$, we can calculate expressions for $C_{i j}, \varepsilon_{l k}$, and $e_{k i}$ in eq 1 as functions of these quantities.

Now we are ready to derive the equations for the piezoelectric constants. From the second, third, and fourth rows in eq 23, putting $E_{i}=0$, $X_{i}{ }^{\nabla}=0$, and $D_{i}{ }^{\nabla}=0$, we obtain

$$
\begin{gathered}
D_{i}=-\phi \varepsilon^{\mathrm{m}} E_{i}^{\mathrm{II}} \\
x_{i}^{\mathrm{II}}=\left(C^{\mathrm{II}}\right)_{i j}^{-1} C_{j k}^{\mathrm{m}} x_{k}+\left(C^{\mathrm{II}}\right)_{i l}^{-1} e_{m l}^{\mathrm{II}} E_{m}{ }^{\mathrm{II}} \\
E_{i}^{\mathrm{II}}=-4 \pi\left(\varepsilon^{\mathrm{II}}\right)_{i j}^{-1} e_{j k}^{\mathrm{II}} x_{k}{ }^{\mathrm{II}}
\end{gathered}
$$

Combining eq 47,48 , and 49 , we obtain

$$
\begin{aligned}
D_{i}= & 4 \pi \phi \varepsilon^{\mathrm{m}}\left[\delta_{i m}+4 \pi\left(\varepsilon^{\mathrm{II}}\right)_{i j}^{-1} e_{j k}^{\mathrm{II}}\left(C^{\mathrm{II}}\right)_{k l}^{-1} e_{m l}^{\mathrm{II}}\right]^{-1} \\
& \times\left(\varepsilon^{\mathrm{II}}\right)_{m n}^{-1} e_{n o}^{\mathrm{II}}\left(C^{\mathrm{II}{ }_{r}}\right)_{o p}^{-1} C_{p_{q}}^{\mathrm{m}} x_{q},
\end{aligned}
$$

where $\delta_{i j}=\left\{\begin{array}{ll}1 & i=j \\ 0 & i \neq j\end{array}\right.$. Then the piezoelectric stress and strain constants of the system are given by

$$
\begin{aligned}
e_{i j}= & D_{i} / 4 \pi x_{j}=\phi \varepsilon^{\mathrm{m}}\left[\delta_{i n}+4 \pi\left(\varepsilon^{\mathrm{II}}\right)_{i k}^{-1} e_{k l}^{\mathrm{II}}\left(C^{\mathrm{II} \mathrm{I}_{\mathrm{r}}}\right)_{l m}^{-1} e_{n m}^{\mathrm{II}}\right]_{i n}^{-1} \\
& \times\left(\varepsilon^{\mathrm{II}}\right)_{n_{0}}^{-1} e_{o p}^{\mathrm{II}}\left(C^{\mathrm{II}}\right)_{p q}^{-1} C_{q j}^{\mathrm{m}}
\end{aligned}
$$

and

$$
\begin{aligned}
d_{i j}= & e_{i j}(C)_{k j}^{-1} \\
= & \phi \varepsilon^{\mathrm{m}}\left[\delta_{i o}+4 \pi\left(\varepsilon^{\mathrm{II}}\right)_{i k}^{-1} \mathrm{~d}_{l i}^{\mathrm{II}} C_{h l}^{\mathrm{II}}\left(C^{\mathrm{II}}\right)_{l m}^{-1} d_{o n}^{\mathrm{II}} C_{n m}^{\mathrm{II}}\right]_{i o}^{-1} \\
& \times\left(\varepsilon^{\mathrm{II}}\right)_{o p}^{-1} d_{p q}^{\mathrm{II}} C_{q r}^{\mathrm{II}}\left(C^{\mathrm{II} \mathrm{r}}\right)_{r s}^{-1} C_{s t}^{\mathrm{m}}(C)_{t j}^{-1}
\end{aligned}
$$

where $d_{k h}^{\mathrm{II}}$ is piezolectric strain constant of the sphere. If the second term in brackets in eq 51 and 52 , which is the piezoelectric secondary effect, can be neglected, we obtain the following simpler relations

$$
\begin{gathered}
e_{i j}=\phi \varepsilon^{\mathrm{m}}\left(\varepsilon^{\mathrm{II}}\right)_{i k}^{-1} e_{k l}^{\mathrm{II}}\left(C^{\mathrm{II}_{\mathrm{r}}}\right)_{l m}^{-1} C_{m j}^{\mathrm{m}} \\
d_{i j}=\phi \varepsilon^{\mathrm{m}}\left(\varepsilon^{\mathrm{II}}\right)_{i k}^{-1} d_{k h}^{\mathrm{II}} C_{h l}^{\mathrm{II}}\left(C^{\mathrm{II}_{\mathrm{r}}}\right)_{l m}^{-1} C_{m n}^{\mathrm{m}}(C)_{n j}^{-1}
\end{gathered}
$$

In these formulas the coefficients $C^{\mathrm{m}}, C^{\mathrm{II}_{\mathrm{r}}}, \varepsilon^{\mathrm{m}}$, and $\varepsilon^{\mathrm{II}_{\mathrm{c}}}$ can be expressed by material constants of phases I and II by eq $40,41,44$, and 45 , respectively.

\section{EXPRESSIONS OF PIEZOELECTRIC CONSTANTS}

In a previous section, we have derived general formulas eq 51 and 52 for the piezoelectric stress constant $e_{i j}$ and the piezoelectric strain constant, $d_{i j}$, for a general spherical dispersion system. If all components of the piezoelectric constant, $e^{\mathrm{II}}$ and $d^{\mathrm{II}}$, the elastic constants, $C^{\mathrm{I}}$ and $C^{\mathrm{II}}$, and the dielectric constants, $\varepsilon^{\mathrm{I}}$ and $\varepsilon^{\mathrm{II}}$, are known, the piezoelectric constants of the system, $\boldsymbol{e}$ and $\boldsymbol{d}$, can be calculated by means of eq 51 and 52 . For the present, we are concerned 
with the effect on the piezoelectric constant of the elastic and dielectric relaxations of the sphere and the medium. Therefore we shall ignore the anisotropy in the piezoelectric, elastic, and dielectric constants and derive some concrete expressions for the piezoelectric constants for three representative examples.

Case I (when the symmetry is $D_{\infty}(\infty 22)$ and the piezoelectricity of the face-shear type only exists in the sphere): This case includes

$$
e_{14}=\phi e_{14}^{\mathrm{II}} \frac{3 \varepsilon^{\mathrm{I}} 5 G^{\mathrm{I}} \frac{K^{\mathrm{I}}+\frac{4}{3} G^{\mathrm{I}}}{K^{\mathrm{I}}+2 G^{\mathrm{I}}}}{\left[2 \varepsilon^{\mathrm{I}}+\varepsilon^{\mathrm{II}}+\left(\varepsilon^{\mathrm{I}}-\varepsilon^{\mathrm{II}}\right) \phi\right]\left[3 G^{\mathrm{I}} \frac{K^{\mathrm{I}}+\frac{8}{9} G^{\mathrm{I}}}{K^{\mathrm{I}}+2 G^{\mathrm{I}}}+2 G^{\mathrm{II}}+2\left(G^{\mathrm{I}}-G^{\mathrm{II}}\right) \phi\right]+8 \pi(1-\phi)^{2}\left(e_{14}^{\mathrm{II}}\right)^{2}}
$$

where $\phi$ is the volume fraction of the dispersed spheres and following changes of notation are made: $C_{44}^{\mathrm{II}} \rightarrow G^{\mathrm{II}}$ and $\varepsilon_{1}^{\mathrm{II}} \rightarrow \varepsilon^{\mathrm{II}}$. The term $8 \pi(1-\phi)^{2}$. $\left(e_{14}^{\mathrm{II}}\right)^{2}$ expresses the piezoelectric secondary effect. If the medium and the sphere are incompressible and the piezoelectric secondary effect is ignored, eq 56 can be simplified as follows

$$
\begin{aligned}
e_{14}= & \phi e_{14}^{\mathrm{II}} \frac{3}{2+\frac{\varepsilon^{\mathrm{II}}}{\varepsilon^{\mathrm{I}}}+\left(1-\frac{\varepsilon^{\mathrm{II}}}{\varepsilon^{\mathrm{I}}}\right) \phi} \\
& \times \frac{5}{3+\frac{2 G^{\mathrm{II}}}{G^{\mathrm{I}}}+2\left(1-\frac{G^{\mathrm{II}}}{G^{\mathrm{I}}}\right) \phi}
\end{aligned}
$$

Similarly, from eq 54 , we can also obtain

$$
\begin{aligned}
d_{14}= & \phi d_{14}^{\mathrm{II}} \frac{3}{2+\frac{\varepsilon^{\mathrm{II}}}{\varepsilon^{\mathrm{I}}}+\left(1-\frac{\varepsilon^{\mathrm{II}}}{\varepsilon^{\mathrm{I}}}\right) \phi} \\
& \times \frac{5}{2+\frac{3 G^{\mathrm{I}}}{G^{\mathrm{II}}}+3\left(1-\frac{G^{\mathrm{I}}}{G^{\mathrm{II}}}\right) \phi}
\end{aligned}
$$

In the above expressions, $e_{14}$ and $d_{14}$ are complex quantities, i.e.,

$$
\begin{aligned}
e_{14} & =e_{14}^{\prime}-i e_{14}^{\prime \prime}=e_{14}^{\prime}\left(1-i \tan \delta_{e}\right) \\
d_{14} & =d_{14}^{\prime}-i d_{14}^{\prime \prime}=d_{14}^{\prime}\left(1-i \tan \delta_{d}\right)
\end{aligned}
$$

because $\varepsilon^{\mathrm{I}}, \varepsilon^{\mathrm{II}}, G^{\mathrm{I}}$, and $G^{\mathrm{II}}$ are all complex quan- most biopolymers and synthetic polypeptides. For simplicity, we assume that $C_{i j}^{\mathrm{II}}=0 \quad(i \neq j$, $i, j=4,5,6)$ and $\varepsilon_{i j}^{\mathrm{II}}=0(i \neq j)$, and that the only nonzero components of $e^{\mathrm{II}}$ are $\pm e_{14}^{\mathrm{II}}$, that is,

$$
\boldsymbol{e}^{\mathrm{II}}=\left[\begin{array}{cccccc}
0 & 0 & 0 & e_{14}^{\mathrm{II}} & 0 & 0 \\
0 & 0 & 0 & 0 & -e_{14}^{\mathrm{II}} & 0 \\
0 & 0 & 0 & 0 & 0 & 0
\end{array}\right]
$$

The calculation according to eq 51 leads to the following formula

tities, which are given as

$$
\begin{gathered}
\varepsilon=\varepsilon^{\prime}-i \varepsilon^{\prime \prime}=\varepsilon^{\prime}\left(1-i \tan \delta_{\varepsilon}\right) \\
G=G^{\prime}+i G^{\prime \prime}=G^{\prime}\left(1+i \tan \delta_{G}\right)
\end{gathered}
$$

From eq 57 and 58, the influence of the elastic and dielectric loss on the phase angle of the piezoelectric constants is expressed as

$$
\begin{aligned}
\tan \delta_{e}= & \frac{1}{1+\frac{2 \varepsilon^{\mathrm{I}}}{\varepsilon^{\mathrm{II}}}}\left(\tan \delta_{\varepsilon}{ }^{\mathrm{I}}-\tan \delta_{\varepsilon}{ }^{\mathrm{II}}\right) \\
& +\frac{2}{2+\frac{3 G^{\mathrm{I}}}{G^{\mathrm{II}}}}\left(\tan \delta_{G}{ }^{\mathrm{II}}-\tan \delta_{G}{ }^{\mathrm{I}}\right) \\
\tan \delta_{d}= & \frac{1}{1+\frac{2 \varepsilon^{\mathrm{I}}}{\varepsilon^{\mathrm{II}}}}\left(\tan \delta_{\varepsilon}{ }^{\mathrm{I}}-\tan \delta_{\varepsilon}{ }^{\mathrm{II}}\right) \\
& +\frac{3}{3+\frac{2 G^{\mathrm{II}}}{G^{\mathrm{I}}}}\left(\tan \delta_{G}{ }^{\mathrm{I}}-\tan \delta_{G}{ }^{\mathrm{II}}\right)
\end{aligned}
$$

Case II (when the elastic constants of the piezoelectric spheres are much larger than those of the medium): This case includes a polarized mixture of piezoelectric ceramic powders, such as PZT, and adhesive polymers. We assume that $\varepsilon_{i j}^{\mathrm{II}}=0(i \neq j), \quad G^{\mathrm{II}}=K^{\mathrm{II}}=\infty, \phi \ll 1$ and that the piezoelectric secondary effect can be ignored. From eq 52 we obtain

$$
\left[\begin{array}{l}
d_{i 1} \\
d_{i 2} \\
d_{i 3} \\
d_{i 4} \\
d_{i 5} \\
d_{i 6}
\end{array}\right]=\phi \frac{3}{2+\frac{\varepsilon_{i}^{\mathrm{II}}}{\varepsilon^{\mathrm{I}}}} \cdot \frac{1+\frac{4}{3} \frac{G^{\mathrm{I}}}{K^{\mathrm{I}}}}{2+\frac{4 G^{\mathrm{I}}}{K^{\mathrm{I}}}}\left[\begin{array}{cccccc}
\beta+4 & \beta-1 & \beta-1 & 0 & 0 & 0 \\
\beta-1 & \beta+4 & \beta-1 & 0 & 0 & 0 \\
\beta-1 & \beta-1 & \beta+4 & 0 & 0 & 0 \\
0 & 0 & 0 & 5 & 0 & 0 \\
0 & 0 & 0 & 0 & 5 & 0 \\
0 & 0 & 0 & 0 & 0 & 5
\end{array}\right]\left[\begin{array}{l}
d_{i 1}^{\mathrm{II}} \\
d_{i 2}^{\mathrm{II}} \\
d_{i 3}^{\mathrm{II}} \\
d_{i 4}^{\mathrm{II}} \\
d_{i 5}^{\mathrm{II}} \\
d_{i 6}^{\mathrm{II}}
\end{array}\right]
$$


where $i=1,2,3, \beta=(4 / 3)\left(G^{\mathrm{I}} / K^{\mathrm{I}}\right)$ and $\varepsilon^{\mathrm{I}}, G^{\mathrm{I}}$, and $K^{\mathrm{I}}$ are the dielectric constant, the shear modulus, and the bulk modulus of the medium, respectively, and $\varepsilon_{i}{ }^{\mathrm{II}}$ the $i$-th component of the dielectric constant of the sphere.

Case III (when the medium and sphere are both incompressible): This case includes semi- crystalline polymers, where piezoelectricity exists in the crystalline phase. One example is polyvinylidene fluoride. We assume that $\varepsilon_{i j}^{I I}=0(i \neq j)$, $K^{\mathrm{I}}=K^{\mathrm{II}}=\infty, \phi \ll 1$, the piezoelectric secondary effect is negligibly small, and the sphere is elastically isotropic. From eq 52, we obtain

$$
\left[\begin{array}{l}
d_{i 1} \\
d_{i 2} \\
d_{i 3} \\
d_{i 4} \\
d_{i 5} \\
d_{i 6}
\end{array}\right]=\phi \frac{3}{2+\frac{\varepsilon_{i}{ }^{\mathrm{II}}}{\varepsilon^{\mathrm{I}}}} \cdot \frac{5 \frac{G^{\mathrm{II}}}{G^{\mathrm{I}}}}{9+6 \frac{G^{\mathrm{II}}}{G^{\mathrm{I}}}}\left[\begin{array}{rrrrrr}
2 & -1 & -1 & 0 & 0 & 0 \\
-1 & 2 & -1 & 0 & 0 & 0 \\
-1 & -1 & 2 & 0 & 0 & 0 \\
0 & 0 & 0 & 3 & 0 & 0 \\
0 & 0 & 0 & 0 & 3 & 0 \\
0 & 0 & 0 & 0 & 0 & 3
\end{array}\right]\left[\begin{array}{l}
d_{i 1}^{\mathrm{II}} \\
d_{i 2}^{\mathrm{II}} \\
d_{i 3}^{\mathrm{II}} \\
d_{i 4}^{\mathrm{II}} \\
d_{i 5}^{\mathrm{II}} \\
d_{i 6}^{\mathrm{II}}
\end{array}\right]
$$

where $i=1,2,3$ and $\varepsilon^{\mathrm{I}}$ and $G^{\mathrm{I}}$ are the dielectric constant and the shear modulus of the medium, respectively, and $G^{\mathrm{II}}$ and $\varepsilon_{i}{ }^{\mathrm{II}}$ are the shear modulus and the $i$-th component of the dielectric constant of the sphere, respectively. Equation 66 includes the expression for $d_{14}$ which has been already given in case $\mathrm{I}$.

Acknowledgment. The author wishes to thank Dr. E. Fukada of the Institute of Physical and Chemical Research for discussion and encouragement throughout this work. He is also grateful to Prof. A. Ookawa of Gakushuin University for valuable discussions.

\section{APPENDIX}

Definitions of notations

$\boldsymbol{X}=$ stress of the system

$\boldsymbol{X}^{\mathbf{v}}=$ virtual stress on the sphere

$f^{\nabla}=$ virtual force on the sphere

$\boldsymbol{x}=$ strain of the system

$\boldsymbol{x}^{\mathrm{II}}=$ strain of the sphere

$\boldsymbol{l}=$ displacement of the surface of the sphere

$\boldsymbol{E}=$ electric field in the system

$\boldsymbol{E}^{\mathrm{II}}=$ electric field in the sphere

$\psi=$ electric potential on the surface of the sphere

$D=$ electric displacement in the system

$D^{\nabla}=$ virtual electric displacement in the sphere

$q^{\nabla}=$ virtual true charge on the surface of the sphere

$C=$ elastic constant of the system

$\boldsymbol{C}^{\mathrm{I}}=$ elastic constant of the medium
$\boldsymbol{C}^{\mathrm{II}}=$ elastic constant of the sphere

$C^{\mathbf{r}}=$ elastic constant of the system when the sphere is rigid $\left(x^{\mathrm{II}}=0\right)$

$\boldsymbol{C}^{\mathrm{m}}=$ elastic coefficient between $\boldsymbol{X}$ and $\boldsymbol{x}^{\mathrm{II}}$ when the system is clamped $(x=0)$ or between $\boldsymbol{X}^{\mathrm{V}}$ and $\boldsymbol{x}$ when the sphere is rigid $\left(\boldsymbol{x}^{\mathrm{II}}=0\right)$

$C^{\mathrm{II}_{\mathrm{r}}}=$ elastic coefficient between $\boldsymbol{X}^{\mathrm{V}}$ and $\boldsymbol{x}^{\mathrm{II}}$ when the system is clamped $(\boldsymbol{x}=0)$

$\varepsilon=$ dielectric constant of the system

$\varepsilon^{\mathrm{I}}=$ dielectric constant of the medium

$\varepsilon^{\mathrm{II}}=$ dielectric constant of the sphere

$\varepsilon^{c}=$ dielectric constant of the system when the sphere is conductive $\left(\boldsymbol{E}^{\mathrm{II}}=0\right)$

$\varepsilon^{\mathrm{m}}=$ dielectric coefficient between $\boldsymbol{D}^{\mathrm{v}}$ and $\boldsymbol{E}$ when the sphere is conductive $\left(\boldsymbol{E}^{\mathrm{II}}=0\right)$ and $\boldsymbol{x}^{\mathrm{II}}=0$, or between $\boldsymbol{D}$ and $\boldsymbol{E}^{\mathrm{II}}$ when the system is shorted $(\boldsymbol{E}=0)$

$\varepsilon^{\mathrm{II}_{\mathrm{c}}}=$ dielectric coefficient between $D^{\mathrm{V}}$ and $E^{\mathrm{II}}$ when the system is shorted $(\boldsymbol{E}=0)$ and $x^{\mathrm{II}}=0$

$\boldsymbol{e}=$ piezoelectric stress constant of the system $e^{\mathrm{II}}=$ piezoelectric stress constant of the sphere

\section{REFERENCES}

1. E. Fukada, Progress in Polymer Science, Japan. (Kodansha) 3, 329 (1972).

2. E. Fukada and M. Date, Polymer J. 1, 410 (1970).

3. R. Hayakawa and Y. Wada, Adv. Polym. Sci., 11, 1 (1973).

4. K. Okano, Rept. Progr. Polym. Phys. Japan, 3, 69 (1960); ibid., 5, 79 (1962).

5. K. W. Wagner, Arch. f. Elektrotechn., 2, 371, 374, 383 (1914). 